This item was submitted to Loughborough's Research Repository by the author.

Items in Figshare are protected by copyright, with all rights reserved, unless otherwise indicated.

\title{
Interactions between cars and motorcycles: testing underlying concepts through integration of on-road and simulator studies
}

PLEASE CITE THE PUBLISHED VERSION

http://drivingassessment.uiowa.edu/

\section{PUBLISHER}

(C) University of lowa

\section{VERSION}

AM (Accepted Manuscript)

\section{PUBLISHER STATEMENT}

This work is made available according to the conditions of the Creative Commons Attribution-NonCommercialNoDerivatives 4.0 International (CC BY-NC-ND 4.0) licence. Full details of this licence are available at: https://creativecommons.org/licenses/by-nc-nd/4.0/

\section{LICENCE}

CC BY-NC-ND 4.0

\section{REPOSITORY RECORD}

Lenne, Michael G., Paul M. Salmon, Vanessa Beanland, Guy H. Walker, Geoffrey Underwood, and Ashleigh J. Filtness. 2019. "Interactions Between Cars and Motorcycles: Testing Underlying Concepts Through Integration of On-road and Simulator Studies". figshare. https://hdl.handle.net/2134/23098. 


\title{
INTERACTIONS BETWEEN CARS AND MOTORCYCLES: TESTING UNDERLYING CONCEPTS THROUGH INTEGRATION OF ON-ROAD AND SIMULATOR STUDIES
}

\author{
Michael G Lenné ${ }^{1}$, Paul M Salmon ${ }^{2}$, Vanessa Beanland ${ }^{3}$, Guy H Walker ${ }^{4}$, Geoff \\ Underwood $^{5}$, and Ashleigh Filtness ${ }^{1}$ \\ ${ }^{1}$ Monash University Accident Research Centre, Monash University, Victoria, Australia \\ ${ }^{2}$ Faculty of Arts and Business, University of the Sunshine Coast, Maroochydore, \\ Queensland, Australia \\ ${ }^{3}$ Research School of Psychology, The Australian National University, Canberra, Australian \\ Capital Territory, Australia \\ ${ }^{4}$ School of the Built Environment, Heriot-Watt University, Edinburgh, Scotland \\ ${ }^{5}$ School of Psychology, University of Nottingham, Nottingham, England \\ Email: Michael.Lenne@monash.edu
}

\begin{abstract}
Summary: We conducted on-road and simulator studies to explore the mechanisms underpinning driver-rider crashes. In Study 1 the verbal protocols of 40 drivers and riders were assessed at intersections as part of a $15 \mathrm{~km}$ on-road route in Melbourne. Network analysis of the verbal transcripts highlighted key differences in the situation awareness of drivers and riders at intersections. In a further study using a driving simulator we examined in car drivers the influence of acute exposure to motorcyclists. In a 15 min simulated drive, 40 drivers saw either no motorcycles or a high number of motorcycles in the surrounding traffic. In a subsequent 45-60 min drive, drivers were asked to detect motorcycles in traffic. The proportion of motorcycles was manipulated so that there was either a high (120) or low (6) number of motorcycles during the drive. Those drivers exposed to a high number of motorcycles were significantly faster at detecting motorcycles. Fundamentally, the incompatible situation awareness at intersections by drivers and riders underpins the conflicts. Study 2 offers some suggestion for a countermeasure here, although more research around schema and exposure training to support safer interactions is needed.
\end{abstract}

\section{OBJECTIVE}

Intersection-based crashes between cars and motorcycles are a significant concern worldwide. Potentially underpinning these crashes are two constructs in the psychological and safety literature: looked-but-failed-to-see (LBFTS) errors and schema (Walker et al., 2011). The former describes the situation whereby a road user directs attention toward the relevant part of the visual scene but fails to process that information. The second concept, schema, provides an explanation for why LBFTS errors occur and refers to the mental templates that guide perception, decision making and action. These schema direct sampling of the environment, which in turn directs behaviour, which in turn modifies schema and so on (e.g. Neisser, 1976).

Recent research suggests that drivers and motorcyclists interpret the same road situations differently (Shahar et al., 2010; Walker et al., 2011), and while such differences are inevitable and indeed appropriate, a degree of compatibility between their situation awareness (SA) is needed to minimise conflicts (Walker et al., 2011). The result of incompatible awareness can 
include LBFTS crashes between cars and motorcycles at intersections (Crundall et al., 2012). The question then becomes how to reduce the occurrence of these failures. Car drivers who are also licenced motorcycle riders are involved in fewer car-motorcycle collisions than car drivers who do not hold a motorcycle licence (Magazzù, Comelli and Marinoni, 2006). A mechanism underlying this effect could be a heightened awareness of motorcycles by drivers with riding experience. One explanation for this is that the drivers with riding experience possess schema that anticipate the presence of riders. Differences in the detection of motorcycles in video-based and simulator studies have been reported between driver groups that do and do not have riding experience (Underwood, In press; Mitsopoulos-Rubens \& Lenné, 2012).

This paper reports on two studies that used on-road and simulator-based methods to extend upon previous research. Study 1 aimed to uncover the nature of driver and rider SA at intersections. Using on-road methods, verbal protocols were collected and network analysis conducted to build SA maps. A driving simulator was then used in study 2 to examine the influence of motorcycle exposure on driver's detection of motorcycles. The key question here was whether short-term exposure to motorcycles would be sufficient to alter drivers' awareness and detection of motorcycles in traffic. Both studies were approved by the Monash University Human Research Ethics Committee.

\section{STUDY 1 - ON-ROAD EXAMINATION OF SITUATION AWARENESS}

\section{Method}

Participants. Twenty drivers (10 males, 10 females) aged 23-62 years $(\mathrm{M}=34.9, \mathrm{SD}=12.53)$ and 18 riders (17 males, 1 female) aged 28-64 years $(\mathrm{M}=45.5, \mathrm{SD}=12.87)$ took part in the study. Participants were recruited through a weekly on-line university newsletter and were compensated for their time and expenses.

On-road route. A $15 \mathrm{~km}$ urban route in Melbourne was used and comprised a mix of arterial roads (50, 60 and $80 \mathrm{~km} / \mathrm{h}$ speed limits) and residential roads (50 km/h speed limit). Seven intersections were selected that included four fully signalised intersections (i.e., all turns controlled by traffic signals), two partially signalised intersections (i.e., some but not all turns controlled by traffic signals), and a non-signalised intersection.

Procedure. Drivers drove the route in an instrumented 2004 Holden Calais sedan equipped to collect various vehicle and driver-related data. A dictaphone was used to record drivers' verbal protocols. Motorcyclists rode the route using their own motorcycle. Each motorcycle was fitted with an Oregon Scientific ATC9K portable camera, which, depending on motorcycle model, was fixed either to the handlebars or front headlight assembly. The ATC9K camera records the visual scene, speed and distance travelled (via GPS). A microphone was fitted inside each rider's motorcycle helmet to record their verbal protocols.

Data analysis. The Leximancer ${ }^{\mathrm{TM}}$ content analysis software and Agna ${ }^{\mathrm{TM}}$ network analysis software were used to describe and analyse participant SA. Leximancer uses text representations of natural language to interrogate verbal transcripts and identify themes, concepts and the relationships between them. The software does this by using algorithms linked to an in-built 
thesaurus and by focussing on features within the verbal transcripts such as word proximity, quantity and salience (Walker et al., 2011). The output is a network representing concepts derived from the verbal transcript and the relationships between them reflected within the verbalisations.

\section{Results}

Figure 1 shows overall networks for drivers and riders at the intersections encountered during the study. Density is a metric which refers to the semantic network as a whole and is a measure of its overall level of interconnectivity (range 0 to 1 ). Higher levels of interconnectivity suggest a richer set of semantic links and a well integrated set of concepts. Analysis showed that driver networks had a higher density than rider networks (0.92 vs 0.84$)$ and also comprised, on average, less concepts than the rider networks (34 vs 37). This indicates that, when negotiating intersections, drivers' SA networks comprise less in terms of knowledge and information from the world but are better connected in terms of the relationships made between concepts (e.g., 'light' is 'green'). This perhaps suggests that the intersection task is simpler for drivers, requiring fewer concepts. The overall riders' network has more concepts but are less connected (i.e., more pieces of information underpinning SA, but they are less connected with one another).
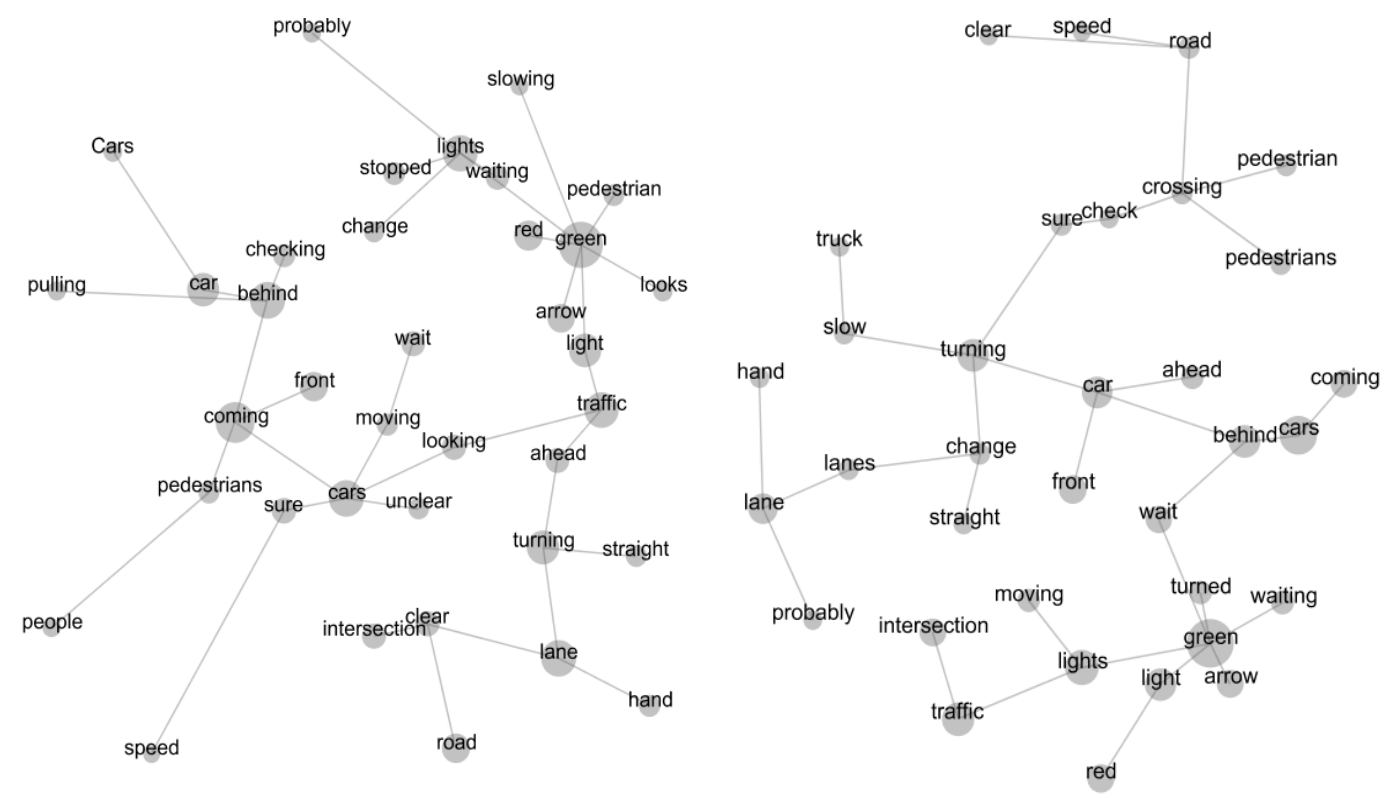

Figure 1. Overall networks for motorcyclists $(n=18$, left $)$ and drivers $(n=20$, right) at intersections

Further inspection reveals that the motorcycle riders have more checking/observation concepts (checking, make sure, looking) than drivers, and the linkage between concepts is interesting. For example, 'checking' is linked to 'behind' in riders but not for drivers. Further, the 'hand' concept, as in right/left-hand side, is present in the rider network but not present in driver network, indicating a potential problem with rider-driver interactions. Overall the analysis provides some evidence that rider SA at intersections is heavily underpinned by checking the environment for other road users and hazards, whereas driver SA is not. 


\section{Rationale for Study 2}

Study 1 has confirmed previous research in demonstrating differences in SA between motorcyclists and drivers. A new finding was that, compared to car drivers, motorcyclists have more concepts that are related to checking for other traffic. This may in part help to explain why car drivers often fail to perceive motorcycles in traffic, since their intersection SA does not incorporate searching the environment for riders. As noted earlier, previous laboratory research has shown that drivers with riding experience have superior abilities to detect motorcycles in traffic compared to car drivers who do not, presumably because these drivers have a schema that contains more elements that prime them to look for and perceive motorcycles in traffic.

Previous studies have manipulated riding experience in car drivers by recruiting participants who possessed very high levels of riding experience. These participants have therefore had many years to accumulate consider levels of exposure to riding in traffic. An interesting research question is to explore the level of riding experience that might be sufficient to confer to measurable benefit to car drivers in how they interact with motorcycles in traffic. Study 2 therefore examined in a driving simulator the influence of 15 min exposure to motorcycles on driver's ability to subsequently detect motorcycles in traffic.

\section{STUDY 2 - SIMULATION-BASED MANIPULATION OF EXPOSURE}

\section{Method}

Participants. Forty licensed drivers with normal or corrected-to-normal visual acuity provided informed consent and received financial compensation (22 female, 18 male; $M=31.9$ years, $S D=7.5)$. The participants drove an average of 10.3 hours per week $(S D=6.6)$ and had held their car licence for an average of 13.1 years $(S D=8.0)$. Drivers were excluded if they had ever held a motorcycle or bus licence to eliminate any potential bias due to experience with these vehicles.

Stimuli. Participants completed two simulator drives. Both drives featured urban roads with one lane in each direction, intersections every $300-500 \mathrm{~m}$, and a $60 \mathrm{~km} / \mathrm{h}(37 \mathrm{mph})$ speed limit. Target vehicle colour (high salience white, low salience grey) and location (left, right, oncoming) were varied in a pseudorandom order, creating six possible variations of each target. The first drive, called the exposure drive, was $7.5 \mathrm{~km}$ (4.7 mi.) long and contained 40 target vehicles. In the "motorcycle" exposure drive all target vehicles were motorcycles and in the "bus" exposure drive all targets were buses. The second drive, called the detection drive, was $39 \mathrm{~km} \mathrm{(24} \mathrm{mi.)}$ long and contained 126 target vehicles. In the "motorcycle" detection drive 120 targets were motorcycles and six targets were buses; in the "bus" detection drive there were 120 buses and six motorcycles. There were equal numbers of each target colour and location.

Procedure. Participants were told the purpose of the study was to examine drivers' behaviour and performance in urban traffic environments while driving in an eca Faros EF-X driving simulator. For the exposure drive participants were instructed to follow the road without turning at intersections and to obey normal road rules including traffic signals and speed limits. They were explicitly instructed to pay attention to the surrounding traffic but were not required to 
detect or identify any specific vehicles. For the subsequent detection drive, in addition to the driving task, participants used two custom-made buttons on the steering wheel to indicate every time they detected a motorcycle or bus. The detection drive lasted approximately an hour with regular breaks. At the conclusion of the study participants were fully debriefed about the experimental aims.

Design. Participants were equally distributed across four experimental conditions. Target prevalence was manipulated between-subjects in a 2 (exposure phase: high motorcycle prevalence, high bus prevalence) $\times 2$ (detection phase: high motorcycle/low bus prevalence, low motorcycle/high bus prevalence) design. The dependent variable of interest here was detection distance (distance between the participant and the target when it was detected). Higher values are equivalent to shorter response times. Distance was used in place of response time because there were no discrete trials; targets moved in and out of view continuously.

\section{Results}

Effects of vehicle type and prevalence. There was no main effect of detection drive on detection distance $(F(1,25)=1.34, \mathrm{p}>0.05)$, but there was a significant main effect of vehicle type $(F(1$, $25)=334.11, \mathrm{p}<.0005)$ and a significant vehicle $\times$ detection interaction $(F(1,25)=208.10$, $\mathrm{p}<.0005)$, indicating an effect of target prevalence in the detection drive. Buses were detected from farther away than motorcycles, but both vehicle types were detected from farther away when they were high prevalence (see Figure 2).

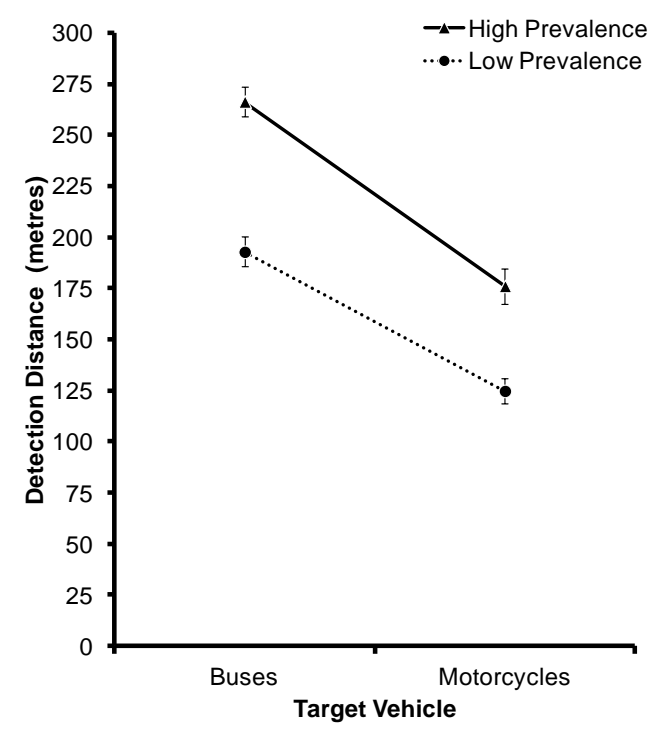

Figure 2. Detection distances for buses and motorcycles as a function of target prevalence in the detection drive. Error bars represent \pm 1 SEM. 


\section{DISCUSSION}

The studies presented here illustrate how on-road and simulator-based methods can be integrated to examine how SA differs on-road between road user groups, and then how potential measures to increase awareness amongst car drivers can be tested.

The first study conducted on-road presents an SA inspired landscape that shows the different networks held by car drivers and motorcyclists. Motorcyclists' SA comprises more elements than for car drivers, and strong themes emerged around motorcyclists checking for surrounding traffic and hazards. While the findings from study 1 are in themselves interesting, and need to be explored further, we were interested also in how to manipulate driver's expectations and behavior around motorcycles.

Study 2 then used simulation to explore whether short-term acute exposure to motorcycles could change drivers' expectations and subsequent detection of motorcycles in traffic. A significant effect of prevalence on the time taken to detect targets was found, with drivers able to detect high-prevalence targets from significantly farther away than low-prevalence targets. This result cannot be attributed to the physical characteristics of targets, since the effect occurred across a range of stimulus varieties, nor to altered scanning patterns due to observers' expectations, since low- and high-prevalence targets appeared in the same locations.

Given their explicit search instructions, our participants should have adopted an attentional set for both buses and motorcycles. It appears that observers' attention was biased towards whichever vehicle was more prevalent during the detection drive, and this bias affected their ability to recognise low-prevalence targets. This is consistent with a recent theory of attentional control, which argued that three factors influence whether a stimulus will capture attention: physical salience, which can capture attention involuntarily; current goals, which voluntarily direct attention based on task demands (i.e., attentional set); and selection history, which prioritises items that have previously been attended or associated with rewards (Awh, Belopolsky, \& Theeuwes, 2012).

In addition to the theoretical implications, our results have practical importance. In particular, they suggest that drivers' difficulty in perceiving motorcyclists is partially due to the fact that motorcycles are relatively rare on our (Australian) roads and consequently drivers do not expect to see them. Drivers' SA therefore may not include riders, which means that their interaction with the world will not anticipate riders. The result of this is drivers not checking the environment for motorbikes and also not expecting to see them, and ultimately, behaving in a manner that is not supportive of safe driver-rider interactions. The findings from the simulator study showed that when motorcycles were high-prevalence, compared to low-prevalence, they were detected on average $51 \mathrm{~m}$ farther away. At a driving speed of $60 \mathrm{~km} / \mathrm{h}$, this allows drivers an extra $3 \mathrm{~s}$ response time. Given that artificially increasing the prevalence of motorcycles on the roads is not a practical solution, in future research it would be worthwhile exploring other methods of eradicating prevalence effects.

Overall, it appears that increasing the prevalence of a visual search target can effectively temporarily increase its salience within the visual environment. The results of the current study 
are consistent with previous research on target prevalence effects, but importantly expand on that research by demonstrating that robust prevalence effects can occur during dynamic and interactive tasks, such as driving, and for tasks where observers do not have to make an explicit present/absent judgement. This research also has important practical implications, since it suggests that the incidence of many perceptual errors while driving may be due to drivers' schema-driven expectations about the types of vehicles that they are most likely to encounter on the road. If we accept that car drivers with a level of riding experience interact more safety with motorcycles in traffic compared to car drivers with no riding experience, and that these benefits are related to more appropriate awareness accumulated through experience and exposure, then a fruitful line of further research is to explore what level of riding experience is necessary to change awareness and improve interactions between cars and motorcycles.

\section{ACKNOWLEDGMENTS}

Study 1 is funded through an Australian Research Council Discovery Grant (DP120100199). Study 2 formed part of the 2-Be-Safe European Union co-funded research project, for which Australian funding was provided through a National Health and Medical Research Council Australian-European Union Collaborative Research Grant (ID 490992). Paul Salmon's contribution to this research is funded through the Australian National Health and Medical Research Council post-doctoral training fellowship scheme.

\section{REFERENCES}

Awh, E., Belopolsky, A.V., \& Theeuwes, J. (2012). Top-down versus bottom-up attentional control: A failed theoretical dichotomy. Trends in Cognitive Sciences, 16, 437-443.

Crundall, D., Crundall, E., Clarke, D., Shahar, A., 2012. Why do car drivers fail to give way to motorcyclists at t-junctions? Accident Analysis and Prevention, 44, 88-96.

Magazzù, D., Comelli, M., Marinoni, A., 2006. Are car drivers holding a motorcycle licence less responsible for motorcycle-car crash occurrence? A non-parametric approach. Accident Analysis and Prevention, 38, 365-370.

Mitsopoulos-Rubens, E., \& Lenné, M.G. (2012). Issues in motorcycle sensory and cognitive conspicuity: The impact of motorcycle low-beam headlights and riding experience on drivers' decisions to turn across the path of a motorcycle. Accident Analysis and Prevention, 49, 86-95.

Neisser, U. (1976). Cognition and reality: principles and implications of cognitive psychology. Freeman, San Francisco.

Shahar, A., Poulter, D., Clarke, D., Crundall, D., 2010. Motorcyclists' and car drivers' responses to hazards. Transportation Research Part F: Traffic Psychology and Behaviour 13, 243-254.

Underwood, G., Ngai, A., \& Underwood, J. (In Press). Driving experience and situation awareness in hazard detection. Safety Science. doi:10.1016/j.ssci.2012.05.025

Walker, G.H., Stanton, N.A., Salmon, P.M., 2011. Cognitive compatibility of motorcyclists and car drivers. Accident Analysis \& Prevention, 43, 878-888. 